\section{Balcón del}

\section{Guadalquivir y} molino de Martos

\section{Córdoba, España}

Córdoba, ubicada en la cuenca del Guadalquivir y que ha sido relevante en diferentes momentos de la historia, es puesta en valor por la generación de espacio público y la remodelación de un antiguo molino de granos. La ribera del río, compuesta por desniveles y abanicos, es la extensión del molino-museo, balcón para admirar la ciudad.

Juan Navarro Baldeweg Doctor Arquitecto, Escuela Técnica Superior de Arquitectura de Madrid FOTOGRAFÍA
Cordoba, located in the basin of Guadalquivir and significant in different moments of history, is given value due to the creation of public space and the remodeling of an ancient grain mill. The riverbank, formed by fanned stepping, is the extension of the mill museum, a balcony for admiring the city. 

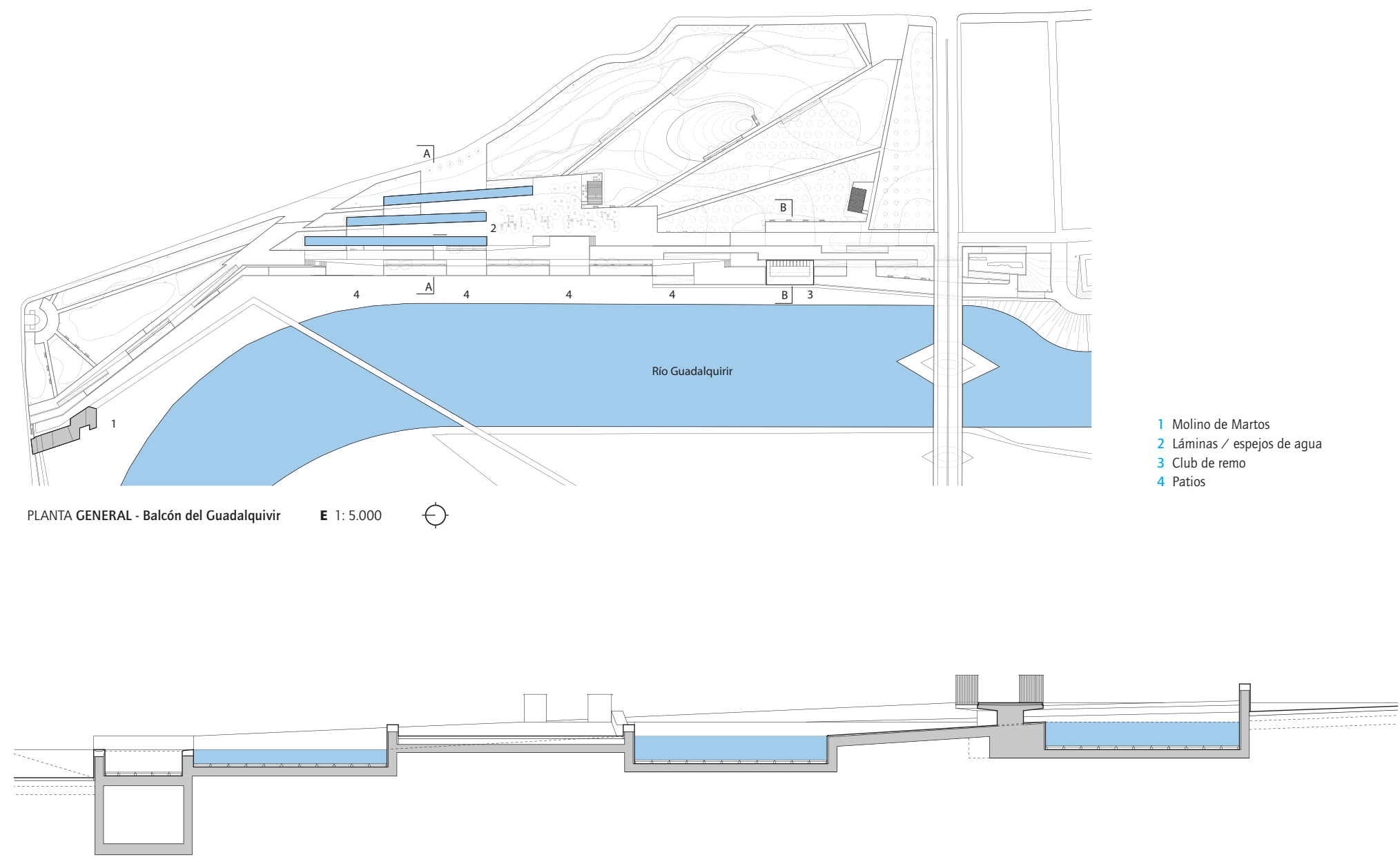

CORTE AA - Láminas / espejos de agua E E 1:250

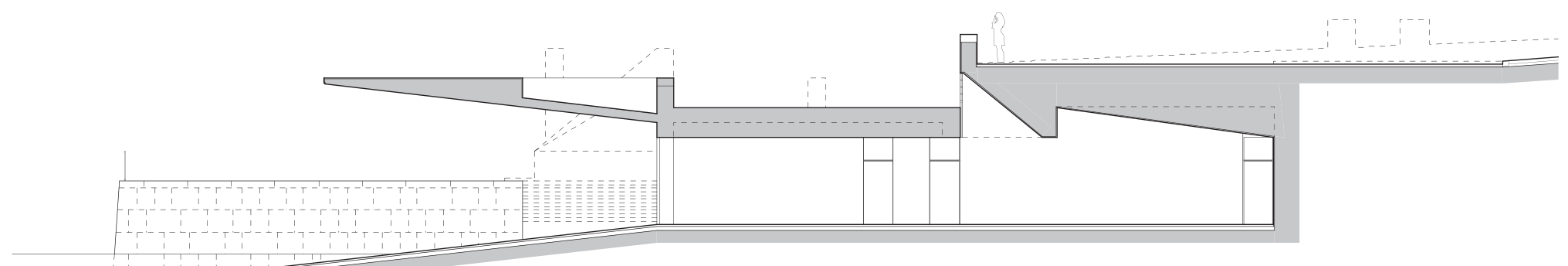

CORTE BB - Club de remo 


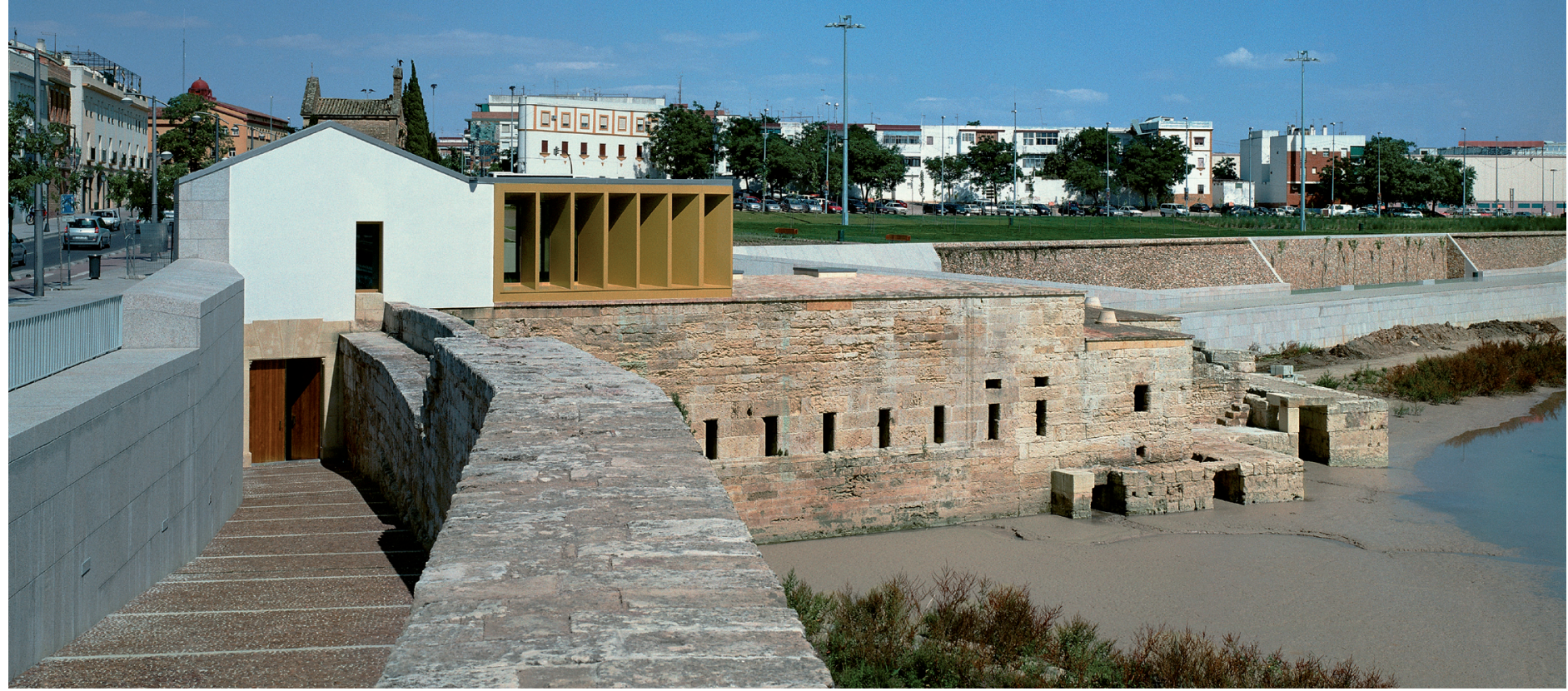

Estos proyectos constituyen una gran intervención urbana en la ciudad de Córdoba, con poderoso efecto sobre la totalidad de ella: se genera un amplio espacio para un parque al borde del río y se rehabilita el molino de Martos, creando un pequeño museo hidráulico que focalizará el espacio público y servirá de enlace entre la ciudad y el parque.

El balcón del Guadalquivir es un lugar que, a causa del giro del curso del río, permite distinguir el perfil de la ciudad antigua, en la que aparece al centro la masa construida de la mezquita-catedral. La situación del molino ayuda a comprender la ciudad y el territorio, el medio construido y el ambiente natural cuya experiencia proporciona el ancho lecho del río.

Formalmente el parque sigue las directrices de tres abanicos que se despliegan desde la Ermita de los Mártires y el molino de Martos hasta el estadio y el puente del Arenal.
El primer abanico está centrado en la ermita, el segundo sirve para organizar entradas por medio de tres estanques conectados que descienden hacia el río y que acercan la experiencia de la proximidad del agua hasta las cotas altas de la ciudad. Entre estas láminas de agua se disponen puentes sobre los planos inclinados por los que se vierte el agua de un estanque a otro. El tercer abanico se abre paso en una amplia zona verde arbolada, dirigiendo los caminos a los distintos puntos de contacto.

La rehabilitación del molino contempla la recuperación de los espacios que albergaban la maquinaria hidráulica en la gran sala abovedada, restaurando cuidadosamente su arquitectura original. Sobre la zona de la sala abovedada se sitúa una sala de exposición del nuevo museo, mirador desde el que se enmarcan vistas al río y a la ciudad. La arquitectura del molino queda así coronada por una pieza realizada en elementos ligeros de aluminio que, apoyándose en la base del cuerpo principal del molino, genera un espacio en el que se funden una geometría reticular y otra radial y en el que se localiza un recinto destinado a la contemplación secuencial y fragmentaria, en un arco de $180^{\circ}$ sobre el río y la ciudad. Este espacio, como una rueda óptica, está articulado y unido por dos ejes verticales en los prismas de cristal que conducen luz cenital sobre la sala abovedada de la planta baja.

El molino y su nuevo mirador exponen de un modo real y metafórico la integración original del molino y el territorio; el nuevo parque y la ciudad antigua. ARQ

\section{Bibliografía sugerida}

AA.V. El Croquis $N^{\circ} 133$. Juan Navarro Baldeweg 1997/2006. El Croquis Editorial, Madrid, 2006. / AA.VV. DA Documentos de Arquitectura $\mathrm{N}^{\circ}$ 56, Juan Navarro Baldeweg. Colegio Oficial de Arquitectura de Almería, España, 2006.

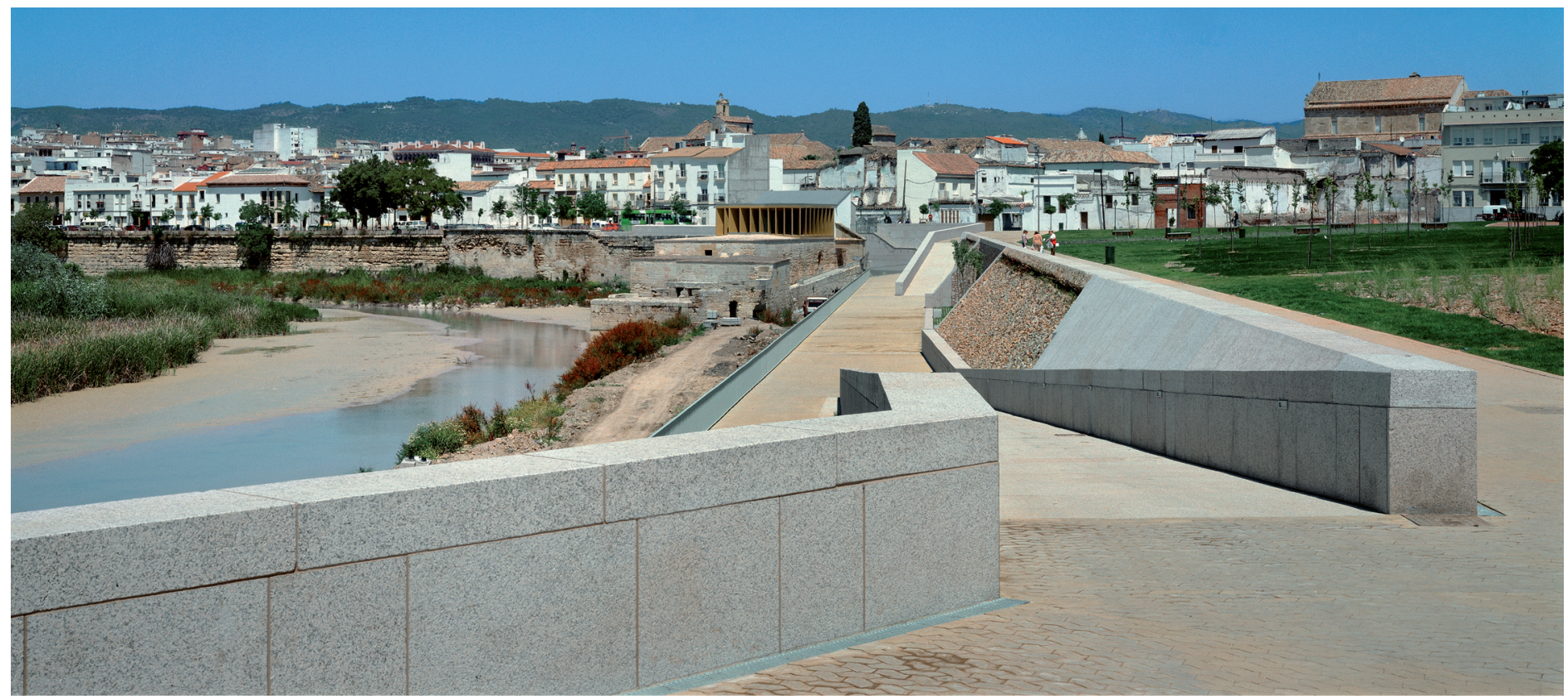




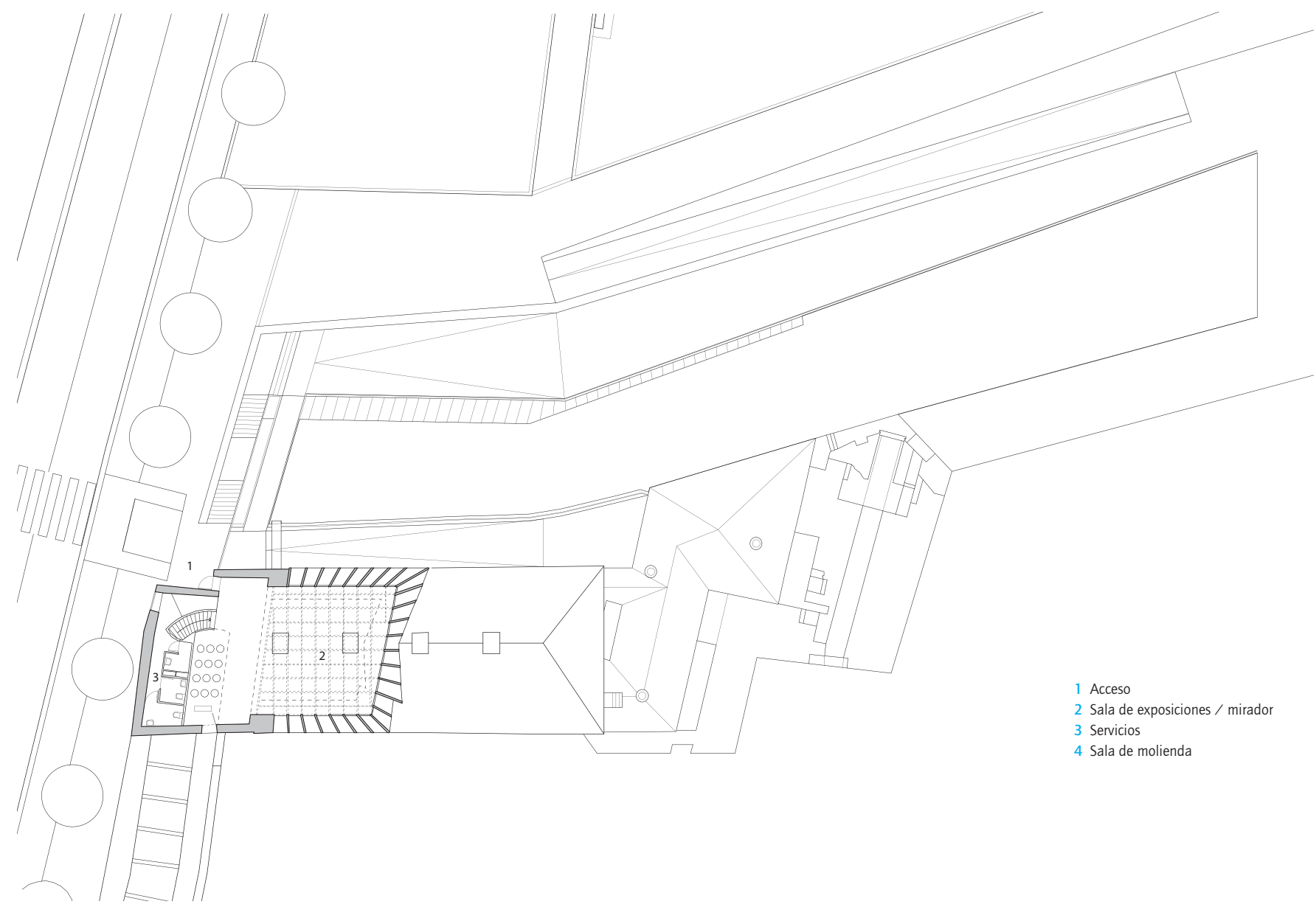

PLANTA NIVEL +-0.00 m Molino de Martos E 1:500

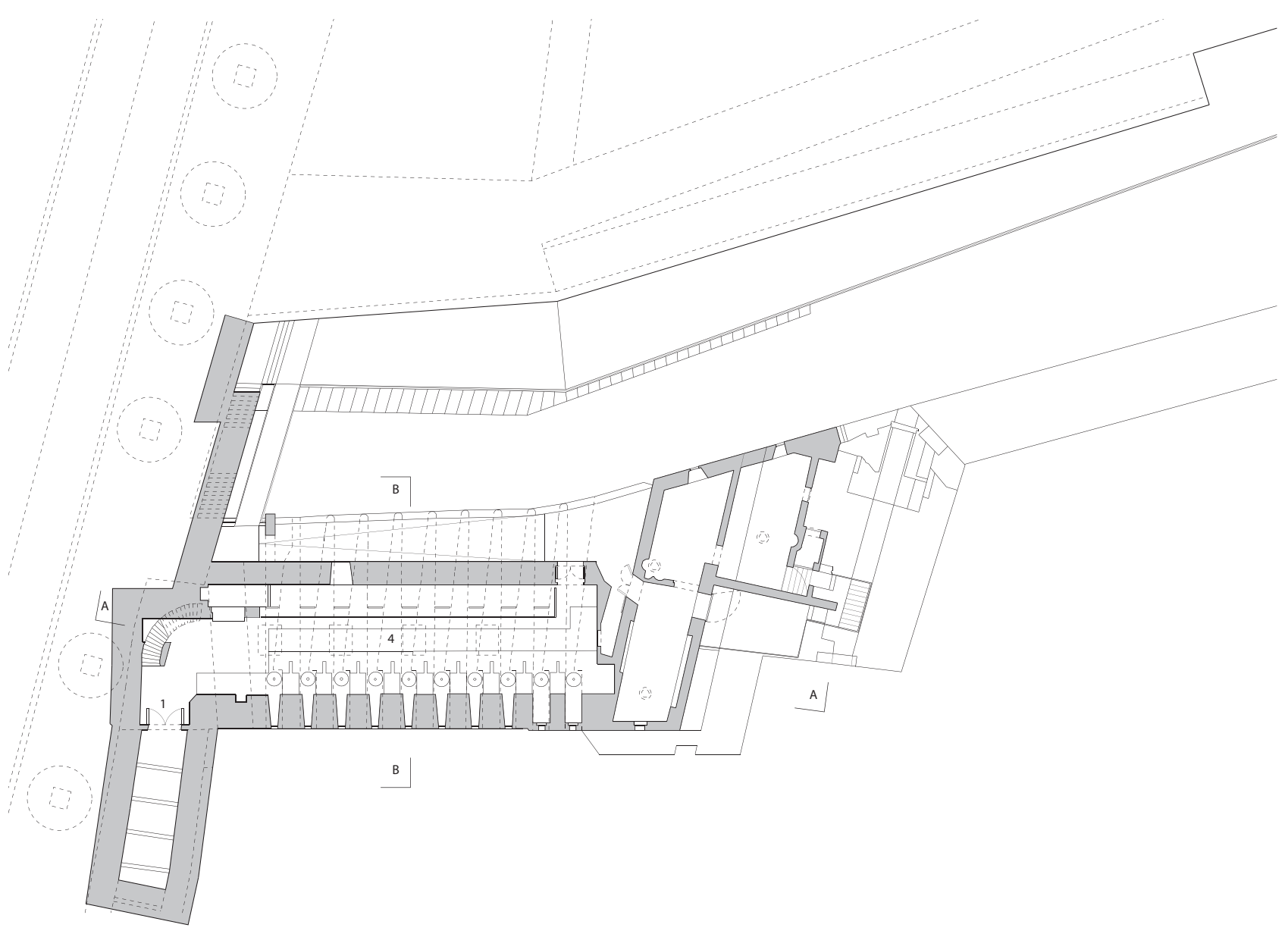



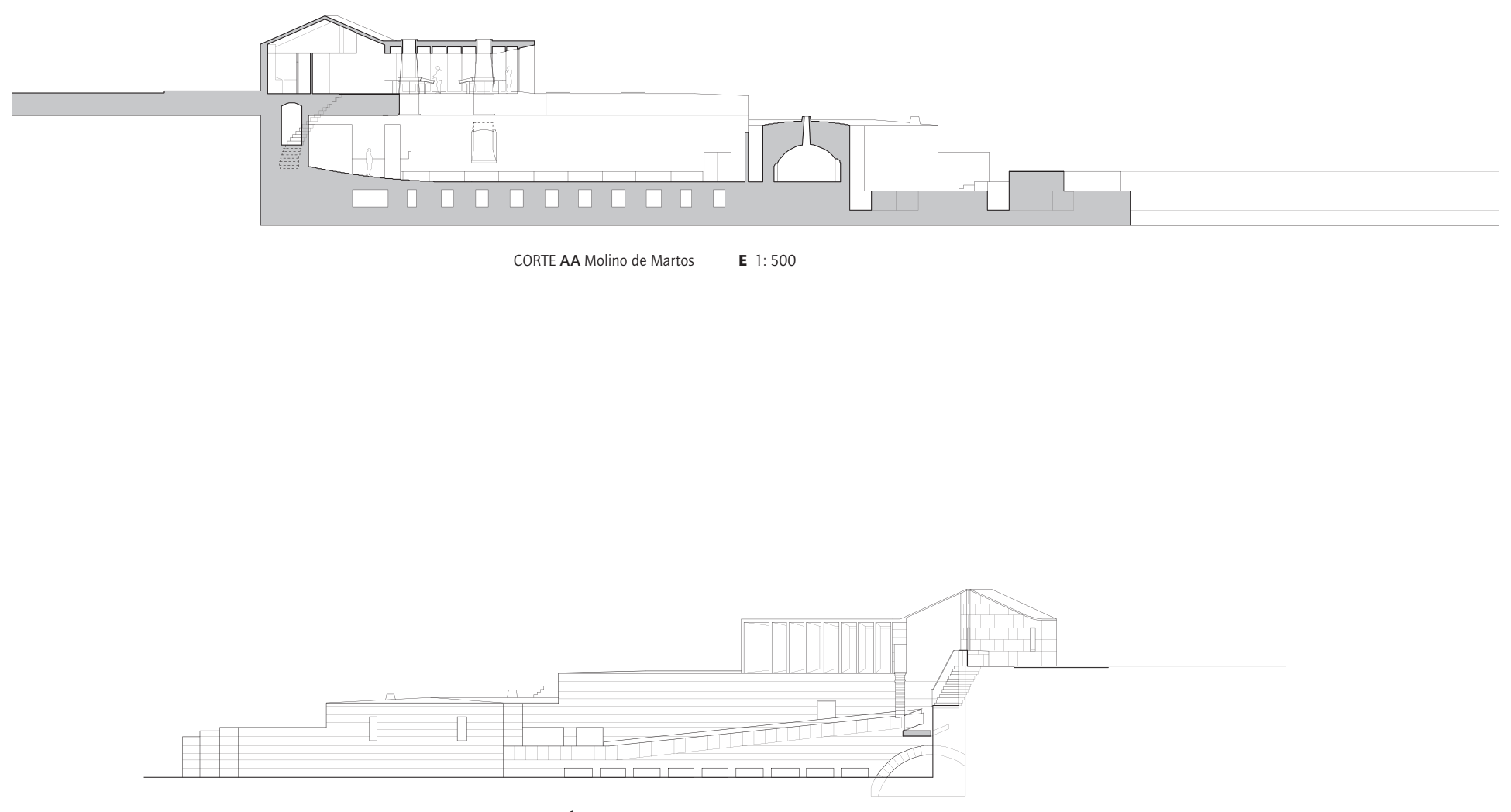

ELEVACIÓN ORIENTE Molino de Martos

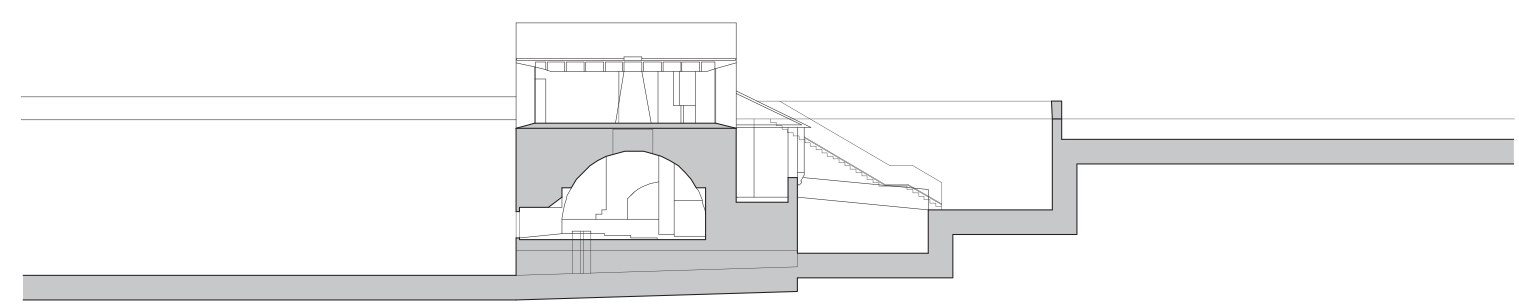

CORTE BB Molino de Martos

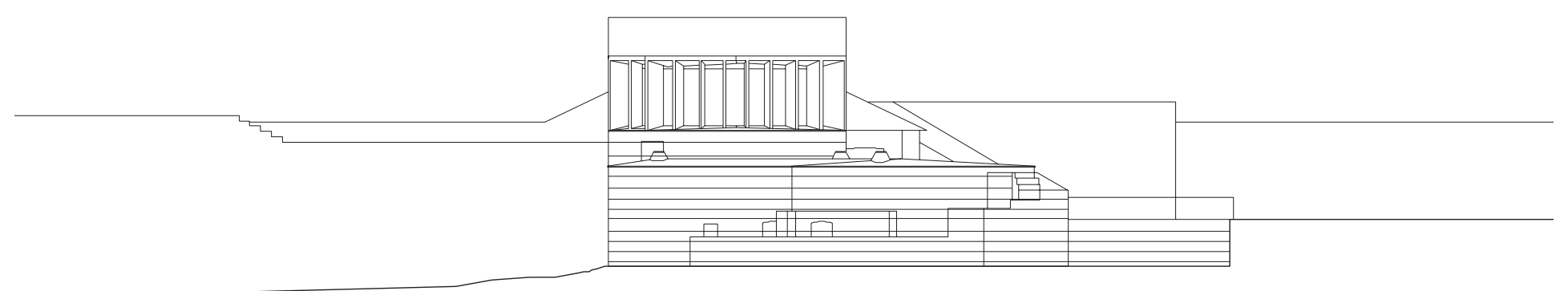

ELEVACIÓN SUR Molino de Martos 
